\title{
ACERCAMIENTO A LA DESMOTIVACIÓN DEL ALUMNADO ADULTO EN EL APRENDIZAJE FORMAL DE LÉXICO DE ESPAÑOL COMO LENGUA EXTRANJERA
}

\author{
APPROACH TO DEMOTIVATION WHILE LEARNING \\ VOCABULARY BY ADULT STUDENTS OF SPANISH AS A FOREIGN \\ LANGUAGE
}

\author{
María del Carmen Méndez Santos \\ Universitat d'Alacant \\ macms@ua.es
}

\begin{abstract}
RESUMEN
La desmotivación es un fenómeno constatado en aulas de lenguas extranjeras en países de todo el mundo y en la mayor parte de los casos los episodios de decaimiento del interés por aprender tienen un vínculo claro con aspectos derivados de la enseñanza formal. Por esta misma razón es necesario analizar el proceso de enseñanza-aprendizaje con el fin de detectar los posibles puntos de mejora. En este trabajo nos centraremos especialmente en la enseñanza del vocabulario porque desde la eclosión de los enfoques léxicos este ha cobrado un papel crucial. Así pues, nos hemos fijado las siguientes preguntas de investigación: (1) ¿Existe la percepción por parte del alumnado adulto de ELE de que el aprendizaje de léxico puede provocar la aparición de episodios de desmotivación? y, de ocurrir, (2) ¿Qué factores son percibidos por el alumnado como desmotivadores? Para lograr respuestas a estas cuestiones hemos empleado un cuestionario con preguntas abiertas y escalas de Likert que fueron analizados en clústeres temáticos, las primeras, y con un análisis factorial, las segundas. Los resultados han demostrado que el aprendizaje de vocabulario sí provoca episodios de desmotivación y que los factores específicos envueltos son el profesorado, exámenes, su dificultad intrínseca, su didáctica, factores psicológicos y afectivos, experiencias de aprendizaje fallidas y los materiales de clase.
\end{abstract}

Palabras clave: desmotivación, español lengua extranjera, enseñanza, léxico, adultos.

\begin{abstract}
Demotivation is a phenomenon found in foreign language classrooms in many researches, and in most cases, those episodes of declining interest in learning have a clear link with aspects derived from formal education. For this reason, it is necessary to analyses the teaching-learning process in order to point out possible issues. In this paper we will focus especially on the teaching of vocabulary because since the emergence of lexical approaches it has taken a crucial role. Therefore, the purpose of this paper is twofold: (1) Is there a perception of adult learners of Spanish as a foreign language that the vocabulary learning can provoke episodes of demotivation? and, if it occurs, (2) What factors are perceived by the students as demotivating? To answer these questions, we have used a questionnaire with open questions and Likert scales that were analyzed in thematic clusters, the former, and with a factor analysis, the latter. The results have shown that vocabulary learning does provoke episodes of demotivation and that the specific factors involved are teachers, exams, its intrinsic difficulty, its didactics, psychological and affective factors, failed learning experiences and class materials.
\end{abstract}

Keywords: demotivation, Spanish as a foreign language, teaching, vocabulary, adults. 


\section{IN'T'RODUCCIÓN}

El aprendizaje de lenguas extranjeras (en adelante, LE) forma parte de la estrategia de numerosos gobiernos e instituciones (Consejo de la Unión Europea, 2008; Gottlieb, 2011; Hu, 2007; Department of Education of the Republic of Philippines, 2012; entre otros muchos) para promover los intercambios financieros en una economía cada vez más globalizada, fomentar la comunicación intercultural, facilitar la movilidad de trabajadores y promover la integración de personas migrantes, entre otras razones.

En muchas ocasiones este interés ha conducido a la introducción de la enseñanza formal de LE en los sistemas educativos, incluso desde edades muy tempranas, como, por ejemplo, recomienda la UE (Comisión Europea, 2011). No obstante, el grado de fracaso a nivel mundial en el estudio de idiomas extranjeros es alto (Dörnyei, 2001; Dörnyei y Ryan, 2015) por lo que conviene reflexionar sobre qué aspectos son mejorables dentro de las políticas lingüísticas que se llevan a cabo.

Desde un punto de vista estructural hay estudios como los de Kaplan, Baldauf y Kamwangamalu (2011) y Baldauf, Kaplan, Kamwangamalu y Bryant (2011) que desentrañan factores que llevan a los sistemas a fallar en sus objetivos como pueden ser la falta de horas de exposición a la lengua meta, la inadecuación de los materiales o la carencia de recursos, las discordancias entre la metodología empleada y los resultados deseados, la falta de formación del profesorado, etc. De hecho, en particular, algunos de estos factores, además de tener implicaciones estructurales, también desencadenan la aparición de episodios de pérdida de interés y desmotivación en el alumnado (Dörnyei, 2001; Kikuchi, 2015). En concreto, en la investigación de Ushioda (1998, citada en Dörnyei, 2001) se constata que casi todos los factores desmotivadores atestiguados estaban relacionados con el modo de aprender las LE mediante la instrucción formal.

En consecuencia, se ha percibido desde la comunidad docente e investigadora la necesidad de analizar qué falla durante la enseñanza de una LE para poder implementar mejoras. Esto ha llevado a que numerosos estudios sobre la desmotivación en el aprendizaje de lenguas extranjeras hayan sido realizados, en 
especial para el inglés ${ }^{1}$. No obstante, la mayoría analizan el fenómeno desde una perspectiva general y son escasos los que investigan la situación particular de la enseñanza de ciertos contenidos o destrezas. Consideramos que esto es necesario para completar el panorama crítico de la desmotivación y así aportar una perspectiva más detallada de todos y cada uno de los factores, contenidos y destrezas implicados en el proceso de enseñanza-aprendizaje.

Es en este sentido donde cobra una especial importancia reflexionar sobre la enseñanza del léxico, en especial dado el creciente protagonismo que ha tomado en la enseñanza en las últimas décadas del siglo XX y primeras del XXI debido al desarrollo de los enfoques léxicos (Richards y Rodgers, 2003; Lewis, 1993; Nation, 2001; Thornbury, 2002; Laufer, 2005; Barcroft, 2015, etc.). De hecho, dentro de los estudios generales de desmotivación que son una referencia, como el de Kikuchi (2015), se establecen categorías específicas dentro de sus análisis cualitativos para la desmotivación provocada por la enseñanza de la gramática o el léxico, aunque sin entrar en detalles pormenorizados y, es por ello por lo que se hace necesario profundizar en estos aspectos. En particular, solamente se ha atestiguado un trabajo centrado principalmente en la desmotivación y el léxico: el de Arefinezhad y Golaghaei (2014) realizado para el inglés como LE, con adultos universitarios en Irán y que se tomará como base para realizar esta investigación.

Enmarcado en este contexto histórico y académico y dada la necesidad de mayor investigación, en este trabajo se analizará, pues, la desmotivación del alumnado adulto durante el aprendizaje de español como lengua extranjera en relación con la enseñanza de léxico.

\section{IAA DESMO'TIVACIÓN}

La desmotivación es un tema de interés desde 1993 si tomamos como referencia la publicación de la investigación de Chambers en The Language Learning Journal, pero cuyo verdadero esplendor tuvo lugar tras los trabajos de Dörnyei (2001), Sakai y Kikuchi (2009) y Kikuchi (2015) que se han convertido en una referencia a nivel

\footnotetext{
${ }^{1}$ Para consultar una revisión de los estudios realizados sobre desmotivación, consúltese Méndez Santos (2019).
} 
mundial. En particular, muchos han sido los estudios que se han escrito para el aprendizaje de inglés como LE en diferentes países y contextos (Muhonen, 2004; Falout y Maruyama, 2004; Arai, 2004; Hasegawa, 2004; Falout y Falout, 2005; Keblawi, 2005; Kaivapanah y Ghasemi, 2011; Shu-Chen, 2012; Li y Zhou, 2013; Xuezheng, 2014; Kim y Kim, 2015; Baba Khouya, 2017; Boonchuayrod y Getkham, 2018, entre otros muchos), pero hay pocos referidos a otras lenguas (Hamada y Grafström, 2014, sobre japonés LE; Bunyamethi, 2018, para el español con aprendices tailandeses y Méndez Santos, 2020).

Como ya se ha explicado, dada la importancia que el vocabulario ha cobrado en la enseñanza-aprendizaje cabría esperar que hubiese más estudios que analizasen los posibles vínculos entre la desmotivación y el aprendizaje de léxico, pero no es así. De hecho, solo se ha encontrado uno dedicado principalmente al tema.

Este fue realizado por Arefinezhad y Golaghaei (2014) en Irán, con adultos que estudiaban en un centro de idiomas mediante una investigación de carácter mixto (entrevistas semiestructuradas y cuestionario). Primero se realizaron las entrevistas para determinar qué factores existían que relacionaban el aprendizaje de léxico y la aparición de episodios de desmotivación. Con esos datos y contando también con la referencia a otros cuestionarios sobre desmotivación previos, diseñaron su propio instrumento. Estos autores incluyeron 65 ítems en forma de escala de Likert, pero no solamente relacionados con el léxico, sino también con el comportamiento del docente, la metodología, la evaluación, etc. En nuestra investigación tomamos como referente ese cuestionario ya validado, pero seleccionando solamente los ítems relacionados con el léxico pues es nuestro foco de interés.

En lo que concierne a la relación entre el aprendizaje de léxico y la desmotivación en el trabajo de Arefinezhad y Golaghaei (2014) se constatan como mayores desmotivadores la falta de recursos, el método y los materiales empleados. Estos resultados concuerdan con lo que ya Falout $(2012$, p. 3) había señalado sobre que la desmotivación "comes not from the method as much as mono-methodical way it is taught, with the learners memorizing by rote huge volumes of words and complicated grammar rules, devoid of context and meaningful application”. También Hu (2011) 
en su estudio sobre la relación entre el grado de desmotivación y el nivel de competencia alcanzados por parte de estudiantes universitarios de inglés de Taiwán, constató que el aprendizaje de vocabulario provocaba la aparición de episodios emocionales negativos.

Por todo lo expuesto y al igual que Afrough, Rahimi y Zarafshan (2014), consideramos que la desmotivación puede ocurrir con respecto no solo a cuestiones generales, sino también con respecto a un contenido o una destreza en particular y, por ello y dados los resultados de investigaciones previas, así como por la gran importancia del léxico en la enseñanza actualmente, el interés por el análisis de la relación entre aprendizaje de vocabulario y desmotivación queda legitimado.

En consecuencia, el objetivo de ese trabajo es reflexionar sobre los posibles vínculos entre la aparición de episodios de desmotivación y el aprendizaje del léxico, para poder ofrecer datos que ayuden a la reflexión de los docentes y la toma de acciones, de ser necesarias, para mejorar la docencia de ELE.

\section{JUSTIIFICACIÓN Y PREGUNTAS DE INVESTIGACIÓN}

Dados los planes gubernamentales e institucionales a nivel internacional para promover el multilingüismo y la importancia que se le otorga al léxico en la didáctica de LE, se ha considerado necesario analizar qué vínculos hay entre su aprendizaje y la aparición de episodios de desmotivación en el alumnado, dado que esta puede desembocar en desinterés e incluso en el abandono de los cursos.

Siguiendo la línea de Arefinezhad y Golaghaei (2014) nos planteamos las siguientes preguntas de investigación: (1) ¿Existe la percepción por parte del alumnado adulto de ELE de que el aprendizaje de léxico puede provocar la aparición de episodios de desmotivación?, y, de ocurrir, (2) ¿Qué factores son percibidos por el alumnado como desmotivadores?

\section{MÉTODO}

Este estudio se plantea como un trabajo de carácter descriptivo y preliminar porque pretende determinar qué aspectos relativos al aprendizaje de léxico que ocurren durante la instrucción formal del español como LE pueden provocar la aparición de episodios de desmotivación en el alumnado. Obviamente los resultados apuntan 
tendencias que no son directamente extrapolables a otros contextos, pero pueden servir para la reflexión de los docentes.

En concreto, este estudio surgió como respuesta a la preparación de un taller de buenas prácticas en la enseñanza de lenguas extranjeras que fue presentado en las $\mathrm{V}$ Jornadas de ELE en Hong Kong en el año 2017. A raíz de aquellos resultados se planeó este estudio preliminar que pretende ser ampliado y mejorado en futuras etapas de investigación. Se combinó una recogida de datos cualitativa y cuantitativa siguiendo la tendencia de trabajos anteriores relacionados con la desmotivación.

\subsection{PERFIL DE LA MUESTRA}

Con el fin de explorar de manera preliminar los posibles factores desmotivadores que emergen durante el aprendizaje de vocabulario de español como LE se eligieron participantes que seguían cursos formales de nivel B2 según el MCER tanto en centros de idiomas de universidades, como de centros privados o academias. La muestra es aleatoria simple.

Los participantes en este estudio fueron 104 de los cuales un 16,3\% eran hombres y el $83,7 \%$ mujeres. La proporción de informantes en función de su edad era la siguiente: $18,3 \%$ menores de 20 años; 78,8\% entre 20 y 30 años y un 2,9\% mayores de 30 años. La media de tiempo que han empleado en aprender español es de 957 días. La mayoría de los participantes estaban inscritos en cursos de español en centros de idiomas universitarios (el 74\%), mientras que el resto lo hacían en academias (26\%). Eran de 14 nacionalidades diferentes con especial presencia de estudiantes chinos.

Se trabajó con participantes que tuviesen un nivel intermedio alto de español por dos razones: (1) porque estos ya han pasado muchas etapas de aprendizaje y son discentes con experiencia por lo que hipotéticamente deberían ser capaces de tener más datos que compartir y, (2) porque había una imposibilidad práctica de traducir el cuestionario a todas las lenguas maternas del alumnado para garantizar la comprensión de las preguntas al 100\%. Se pretendía aplicar el instrumento de forma digital, pero dada la censura en China ante ciertas aplicaciones y los problemas que parecía que se planteaban, se optó por distribuirlo en formato papel. 
Se llegó a conformar la muestra, que como se ha dicho es aleatoria simple, después de pedir la colaboración en redes sociales de profesorado de ELE. Quienes se ofrecieron como voluntarios facilitaron el cuestionario a su estudiantado y, o bien los enviaban escaneados a la investigadora, o bien lo hacía el propio alumnado al correo electrónico que se facilita en el mensaje de ética de la investigación que se presenta al comienzo del cuestionario (Rasinger, 2019).

Se facilitó asimismo al profesorado de los cursos un cuestionario para triangular las respuestas contrastando métodos, actividades y técnicas de enseñanza de léxico con las experiencias del alumnado y, así, poder entender mejor el fenómeno, pero desafortunadamente no hubo respuesta de todas las personas implicadas con lo que de los siete docentes solamente tres lo contestaron. La muestra es pues en este aspecto limitada y no se tendrán en cuenta los resultados. Para el futuro debe trabajarse mejor este aspecto, posiblemente empleando otros instrumentos como entrevistas semiestructuradas o haciendo trabajos más ajustados a un contexto específico y no tan generalistas.

\subsection{INSTRUMENTO}

El cuestionario que se distribuyó entre el alumnado fue diseñado teniendo en cuenta trabajos anteriores como el de Arefinezhad y Golaghaei (2014), pero ciñéndose estrictamente a aspectos relativos al léxico y considerando las respuestas que se habían conseguido para la elaboración del taller de buenas prácticas que fue presentado en las V Jornadas de ELE en Hong Kong en 2017. En aquella ocasión se recogieron datos de la desmotivación por parte del alumnado de ELE en general y de entre todas las respuestas se seleccionaron los aspectos que estaban relacionados con el aprendizaje de léxico. Con todos ellos y con base al cuestionario validado del trabajo de Arefinezhad y Golaghaei (2014) se redactaron los 15 ítems en forma de escala Likert.

Este cuestionario de investigación comenzaba con un mensaje de presentación que se corresponde con las normas de buenas prácticas de investigación (Rasinger, 2019). En él se hacen constar el nombre de la investigadora y un correo de contacto y se notifica a los informantes que sus datos serán tratados de forma anónima y con fines estrictamente de carácter investigador. 
Asimismo, se siguieron las fases recomendadas para validar el constructo (Perry, 2017). Por ello, en primer lugar, se contó con la revisión de profesorado e investigadores de ELE, en concreto de dos expertos en léxico y su enseñanza, que asesoraron sobre el formato y la redacción de las preguntas para obtener unos datos de calidad. De hecho, de la primera versión a la versión final, se tuvieron en cuenta modificaciones en los enunciados de las preguntas y en el número de ítems del cuestionario. También se añadieron ejemplos de frases y estructuras modelo en la parte del informe personal para ayudar al alumnado a entender el cuestionario. Esto también fue comprobado. Así, siguiendo las recomendaciones de Brown (2001, p. 62) el cuestionario fue pilotado con 10 estudiantes.

El cuestionario finalmente se dividió en tres partes: La primera, centrada en datos sociodemográficos de respuesta múltiple (sexo, edad, tiempo de estudio del español, nacionalidad); la segunda, un informe personal y reflexivo del alumnado, en forma de pregunta abierta; y la tercera, el cuestionario de 15 ítems con escalas de Likert.

Las respuestas de la primera parte se emplearon para dibujar el perfil de la muestra.

La segunda parte del informe personal y reflexivo del alumnado era una pregunta abierta, sin límite de espacio formulada como sigue: "¿Qué cosas te resultan más motivadoras y desmotivadoras de APRENDER EL LÉXICO cuando estás estudiando una lengua extranjera? (Pon ejemplos y explica tus respuestas, gracias) Por ejemplo: Me gustan las actividades de léxico porque... / Aprendo mucho léxico si... / Las actividades que más me gustan... / Estudio léxico haciendo...”. Las respuestas de esta pregunta abierta han sido analizadas con el programa QDA Miner Lite, de modo que se trazaron clústeres temáticos categorizados mediante etiquetas. Con esta pregunta se pretendía saber si la experiencia aprendiendo léxico era positiva o no, qué cosas gustaban más y menos, sin sesgarles, dando la oportunidad al alumnado de responder libre y abiertamente.

La tercera parte del cuestionario se correspondía con la batería de 15 ítems (véase tabla 1) basada en el trabajo de Arefinezhad y Golaghaei (2014), pero escogiendo solamente aquellos relacionados con el léxico. Se tuvo en cuenta, además, las revisiones de las personas expertas y los resultados del taller de Hong Kong para terminar de definirlos. Estos se plantearon en forma de escala de Likert de 5 puntos 
DESMOTIVACIÓN DEL ALUMNADO ADULTO EN EL APRENDIZAJE FORMAL DE LÉXICO DE ESPAÑOL

(1. Totalmente en desacuerdo, 2. En desacuerdo, 3. Ni de acuerdo ni en desacuerdo, 4. De acuerdo, 5. Totalmente de acuerdo). Por lo tanto, cuanto más alto fuese el grado de acuerdo, mayor fuerza presentaba el factor desmotivador. Las respuestas fueron guardadas y los datos importados a un documento de Excel para su análisis.

\begin{tabular}{|c|c|}
\hline Cód. & Ítems \\
\hline AF1 & En cada clase se introduce demasiado vocabulario \\
\hline AF2 & El vocabulario no se explica tanto como la gramática \\
\hline AF3 & El vocabulario se reutiliza pocas veces y se me olvida \\
\hline AF4 & Necesito más tareas de refuerzo de vocabulario \\
\hline AF5 & Me estresa tener que estudiar vocabulario \\
\hline AF6 & Las listas de vocabulario no son útiles para mí \\
\hline AF7 & El vocabulario que estudiamos no es útil \\
\hline AF8 & Tengo pocas estrategias para aprender vocabulario nuevo \\
\hline AF9 & $\begin{array}{l}\text { Me frustro cuando no puedo entender todas las palabras de un } \\
\text { texto }\end{array}$ \\
\hline AF10 & El vocabulario que aprendo no es interesante \\
\hline AF11 & El vocabulario que aprendo no es relevante para mí \\
\hline AF12 & $\begin{array}{l}\text { No he encontrado la mejor manera para mi estilo para aprender } \\
\text { vocabulario }\end{array}$ \\
\hline AF13 & Las actividades de vocabulario no me parecen divertidas \\
\hline AF14 & Las actividades de vocabulario no me hacen sentir que aprendo \\
\hline AF15 & Hago demasiadas tareas de vocabulario que no me parecen útiles \\
\hline
\end{tabular}

\subsection{PROCEDimiento}

El alumnado que contestó este cuestionario fue partícipe de forma voluntaria y su profesorado le facilitó tiempo durante las clases para poder rellenarlo y también les ofreció la posibilidad de rellenarlo fuera del aula y enviarlo directamente a la investigadora responsable. El propio profesorado dio una explicación de la investigación y contestó preguntas sobre cómo contestar el cuestionario. Estas instrucciones fueron proporcionadas por la investigadora previamente.

Los cuestionarios que llegaron vacíos o parcialmente contestados fueron descartados. El total de respuestas válidas fue de 104. El tiempo de recogida de la 
muestra fue entre abril y julio de 2018. La media de duración que se empleó para responderlo fue de entre 20 y 30 minutos.

\subsection{LiMITACIONES}

Al ser un primer acercamiento se pretendía solamente realizar una aproximación al tema y comprobar si había algún patrón que pudiese ser de interés para seguir analizando la posible relación entre enseñanza de léxico y la desmotivación en posteriores estudios. Por ello no se pretendía lograr una cantidad alta de respuestas, de hecho, la muestra es pequeña, ya que cuenta con 104 informantes.

Además, como la recogida de datos estaba condicionada al número de profesores que se ofrecieran a cooperar, la muestra resultó tener un perfil muy heterogéneo, aunque sí es posible decir que todo es alumnado adulto de ELE que sigue un curso formal de nivel B2.

Por supuesto, el hecho de que unos docentes se hayan ofrecido a colaborar puede esconder una especial sensibilidad por el tema y la didáctica de léxico, con lo que, podría intuirse, sus clases son especialmente cuidadosas en el tema de cómo enseñarlo. Por una parte, esto condiciona la generalización de las respuestas de este trabajo, pero también tiene un aspecto paradójico: a pesar de que los docentes (lo sabemos especialmente por los tres informes de triangulación que sí redactaron) estaban técnicamente concienciados con la importancia del léxico, las respuestas de los estudiantes siguen dejando traslucir las mismas quejas que se habían atestiguado en la preparación del taller de las Jornadas de Hong Kong.

Para el futuro, y una vez constatado que sí existe una relación entre aprendizaje de léxico y la aparición de episodios de desmotivación, sería recomendable replicar el estudio cambiando la parte dos del informe personal del alumnado por entrevistas semiestructuradas, ya que muchas respuestas fueron muy cortas.

\section{RESULTADOS Y ANÁLISIS}

\subsection{EL INFORME PERSONAL}

Las respuestas de la pregunta abierta (¿Qué cosas te resultan más motivadoras y desmotivadoras de APRENDER EL LÉXICO cuando estás estudiando una lengua extranjera?) de la parte II denominada Informe personal, fueron analizadas usando el programa QDA Miner Lite. 
En primer lugar, la investigadora principal digitalizó todas las respuestas en un documento para cargarlo al programa. Analizó las mismas buscando clústeres temáticos consistentes. Creó una serie de etiquetas para clasificar las respuestas y las aplicó. Una vez realizado este paso, se envió el archivo de datos a otro investigador que ya había colaborado también en la supervisión de la redacción del cuestionario en general. Se buscaba comprobar que los clústeres estaban bien determinados y que guardaban la precisión necesaria. Para ello se le hizo llegar a este investigador la información sin clasificación ni categorías ya determinadas para ver si llegaba a determinar las mismas categorías y marcar las mismas respuestas como ejemplos de los factores desmotivadores. El etiquetaje de respuestas sobre aspectos desmotivadores fue idéntico. La nomenclatura que empleó el investigador de control no era exactamente -y lógicamente- a la que se ha encontrado en otros trabajos de investigación sobre el tema, pero los fenómenos representados eran los mismos como se detalla más adelante.

A continuación (véase tabla 2), se ofrece una descripción de los factores desmotivadores relacionados con el aprendizaje de léxico atestiguados en el informe personal del alumnado de respuesta abierta. En total se documentaron 30 comentarios sobre estos aspectos. Los comentarios positivos -que los había- quedan excluidos explícitamente de este trabajo porque queremos centrarnos en los aspectos desmotivadores. Estos fueron etiquetados bajo los siguientes códigos: Profesorado, Exámenes, Dificultad, Didáctica y Factores psicológicos y afectivos. Hemos procurado respetar en la medida de lo posible la máxima transparencia de significado de las etiquetas a la vez que hemos procurado usar nombres ya empleados en trabajos previos sobre desmotivación. En la tabla 2 también se ofrece la descripción de qué tipo de testimonios recoge cada código. Asimismo, se ofrece el número de menciones a cada uno de ellos y su porcentaje con respecto a la muestra. 


\begin{tabular}{|c|c|c|c|c|}
\hline \multirow{6}{*}{$\begin{array}{c}\text { Factores } \\
\text { desmotivadores }\end{array}$} & Código & Descripción & $\begin{array}{c}\text { Menciones } \\
(n=30)\end{array}$ & \% Códigos \\
\hline & Profesorado & $\begin{array}{l}\text { Aspectos relacionados con la falta } \\
\text { de claridad del profesorado a la } \\
\text { hora de explicar las palabras }\end{array}$ & 1 & $0,5 \%$ \\
\hline & Exámenes & Las pruebas en sí mismas & 1 & $0,5 \%$ \\
\hline & $\begin{array}{l}\text { Dificultad } \\
\text { intrínseca del } \\
\text { aprendizaje de } \\
\text { vocabulario }\end{array}$ & $\begin{array}{l}\text { Aspectos relacionados con la } \\
\text { dificultad cognitiva de aprender } \\
\text { tantas variaciones, sinónimos, } \\
\text { recordarlas... }\end{array}$ & 4 & $2,1 \%$ \\
\hline & Didáctica & $\begin{array}{l}\text { Aspectos relacionados con la gran } \\
\text { cantidad de ejercicios de } \\
\text { vocabulario -en muchas } \\
\text { ocasiones descontextualizados-, } \\
\text { la memorización de listas de } \\
\text { palabras, la poca falta de } \\
\text { relevancia para sus intereses } \\
\text { personales, la gran cantidad de } \\
\text { palabras a la vez que se espera que } \\
\text { aprendan, etc. }\end{array}$ & 15 & $7,9 \%$ \\
\hline & $\begin{array}{l}\text { Factores } \\
\text { psicológicos y } \\
\text { afectivos }\end{array}$ & $\begin{array}{l}\text { Aspectos relacionados con los } \\
\text { sentimientos que les provoca no } \\
\text { entender, aprender o recordar el } \\
\text { vocabulario (estrés, ansiedad, } \\
\text { desinterés, desesperanza, } \\
\text { frustración...), }\end{array}$ & 9 & $4,7 \%$ \\
\hline
\end{tabular}

Tabla 2. Codificación en clústeres temáticos de las respuestas abiertas del informe del alumnado sobre la pregunta 2: ¿Qué cosas te resultan más motivadoras y desmotivadoras de APRENDER EL LÉXICO cuando estás estudiando una lengua extranjera?

Como es posible observar en la tabla 2, el alumnado experimenta episodios específicos de desmotivación relacionados con el aprendizaje de vocabulario, al igual que se dejaba entrever en anteriores investigaciones más generalistas. Dado este resultado es posible afirmar que el primer objetivo de investigación de este trabajo queda cumplido.

De entre los factores desmotivadores constatados destacan con particular relevancia aquellos relacionados con aspectos didácticos y afectivos. Esto encaja perfectamente con las apreciaciones de Falout (2012, p. 3) que recuerda que la desmotivación no deviene directamente del empleo de un método, sino de que solo se enseñe siempre de la misma manera y también de la memorización de grandes cantidades de palabras sin más. Ello provoca unos sentimientos en el alumnado de indefensión, de sensación de falta de estrategias de aprendizaje, de sentirse sobrepasados y poco capacitados para el aprendizaje. Esto concuerda con las 
apreciaciones de $\mathrm{Hu}$ (2011) sobre que los estudiantes se sentían como "malos aprendiendo vocabulario".

\subsection{El CUESTIONARIO}

Antes de proceder a realizar el análisis factorial, se comprobó las asunciones para el método (Tabachnick y Fidell, 2007) en términos de normalidad, linearidad, valores atípicos univariantes y multivariantes, ausencia de multicolinealidad y singularidad, y factorabilidad de First, para lo que se comprobamos si había muchos valores perdidos dentro de los casos. Después de la primera verificación, el número total de casos potencialmente analizables fue de 104. Posteriormente, buscamos valores atípicos. Dado que estos tienen un efecto drástico en el análisis, queríamos asegurarnos de que no teníamos ninguno dentro de nuestra muestra.

Para identificar los valores atípicos multivariantes, calculamos la distancia de Mahalanubis y verificamos el valor p del Chi-Cuadrado. En los casos en que el valor p esté por debajo de 0,001, el caso debe eliminarse del conjunto de datos. En nuestro conjunto de datos, todos los casos estaban por encima del umbral de 0,001 por lo que los 104 casos podían ser analizados.

Al haber completado los dos supuestos iniciales del conjunto de datos, identificar los valores perdidos y los valores atípicos, procedemos a la verificación de la normalidad. Según la prueba de Kolmogorov-Smirnov, todas las variables no se distribuyeron normalmente. A pesar de que el control de normalidad no se cumplió, podemos continuar con análisis factorial. Como señalaron Muthen y Kaplan (1992) en el caso de datos no distribuidos normalmente, los mínimos cuadrados generalmente muestran índices realistas de ajuste general y valores de parámetros menos sesgados. En esa línea seguimos la recomendación de Olson et al. (2000), quienes notaron que los métodos de estimación de máxima probabilidad y mínimos cuadrados ponderados funcionan mejor con muestras grandes ( $\mathrm{N}=1000$ o más), ya que nuestra muestra es de 104 participantes, decidimos adoptar un método de estimación de mínimos cuadrados.

Además, para justificar la adecuación de la muestra para el análisis factorial, estimamos la Medida de adecuación de muestreo (KMO) de Kaiser-Meyer Olkin, que 
debe ser superior a 0,6 y el valor de la prueba de Esfericidad de Bartlett, que debe ser estadísticamente significativo. Ambos criterios se cumplieron en la muestra, KMO igual a 0.786 y la prueba de Esfericidad de Bartlett es estadísticamente significativa, lo que confirmaba que sí era factible proceder con el análisis factorial. Además, la varianza total explicada cumple los criterios de ser superior a 0,5 (Hair et al. 1999, p. 639). Los coeficientes de fiabilidad variaron de .657 a .749, lo que indica consistencias internas aceptables (ver Field, 2005).

Para estimar el número de factores que se extraerán, tomamos en consideración los factores con valores propios iniciales superiores a 1,1. La cantidad total de ítems considerados es de siete agrupados en dos factores que representan el 58,347\% de la variación (Véase tabla 3).

\begin{tabular}{|c|c|c|}
\hline Código & Ítems & F2 \\
\hline & \multicolumn{2}{|c|}{$\begin{array}{l}\text { Factor 1: Experiencia de aprendizaje } \\
\text { (Cron.alpha=0,749) }\end{array}$} \\
\hline rAF5 & $\begin{array}{l}\text { Me estresa tener que estudiar } 0,550 \\
\text { vocabulario }\end{array}$ & \\
\hline rAF8 & $\begin{array}{l}\text { Tengo pocas estrategias para } 0,566 \\
\text { aprender vocabulario nuevo }\end{array}$ & \\
\hline rAF11 & $\begin{array}{l}\text { El vocabulario que aprendo no es } \quad 0,624 \\
\text { relevante para mí }\end{array}$ & \\
\hline \multirow[t]{2}{*}{ rAF12 } & $\begin{array}{l}\text { No he encontrado la mejor } 0,768 \\
\text { manera para mí para aprender } \\
\text { vocabulario }\end{array}$ & \\
\hline & Factor 2: Materiales de clase (Cron.alp & $=0,657)$ \\
\hline rAF13 & $\begin{array}{l}\text { Las actividades de vocabulario no } \\
\text { me parecen divertidas }\end{array}$ & 0,693 \\
\hline rAF14 & $\begin{array}{l}\text { Las actividades de vocabulario no } \\
\text { me hacen sentir que aprendo }\end{array}$ & 0,583 \\
\hline rAF15 & $\begin{array}{l}\text { Hago demasiadas tareas de } \\
\text { vocabulario que no me parecen } \\
\text { útiles }\end{array}$ & 0,528 \\
\hline
\end{tabular}

Tabla 3. Agrupación de factores significativos como resultado del análisis factorial

Para examinar si los dos factores difieren en términos de las respuestas de los participantes, se calcularon las puntuaciones medias de los ítems que se constataron en los dos factores y se compararon mediante un análisis de varianza de medidas repetidas unidireccional (ANOVA) (Véase tabla 4). 
No hubo una diferencia estadísticamente significativa entre los grupos según lo determinado por ANOVA unidireccional $(p=0,057)$. Según la homogeneidad de la prueba de varianza (la prueba de Levene fue superior a 0,5), lo que implica que no se violó la homogeneidad de la varianza. Una prueba post hoc de Tukey reveló que, independientemente de la edad de los participantes, los factores desmotivadores eran igualmente importantes para desmotivar al estudiante al aprender el vocabulario.

\begin{tabular}{cccccc}
\hline Factor & K & Sign. & $\begin{array}{c}\text { Desviación } \\
\text { típica }\end{array}$ & Asimetría & Curtosis \\
\hline $\begin{array}{c}\text { F1: } \\
\begin{array}{c}\text { Experiencia de } \\
\text { aprendizaje }\end{array}\end{array}$ & 4 & 2,82 & 0,757 & $-0,171$ & $-0,344$ \\
\hline $\begin{array}{c}\text { F2: Materiales } \\
\text { de clase }\end{array}$ & 3 & 2,48 & 0,707 & 0,302 & 0,169 \\
\hline
\end{tabular}

Nota: El error estándar de asimetría es 0.237; el de la curtosis es 0.469

Tabla 4. Resultados del análisis ANOVA

El primer factor fue denominado experiencia de aprendizaje, dado que los ítems significativos (Me estresa tener que estudiar vocabulario, Tengo pocas estrategias para aprender vocabulario nuevo, El vocabulario que aprendo no es relevante para mí, No be encontrado la mejor manera para mi para aprender vocabulario) reflejan la percepción subjetiva y los sentimientos del alumnado sobre su práctica como discentes desde el punto de vista del proceso. Podría considerarse que entronca con parte de lo que Dörnyei (2001) denominó en su trabajo sobre la desmotivación, Reduced self-confidence (Experience of failure or lack of success). También Kikuchi (2015) categorizó la etiqueta Experiences of failure, dentro de la que registró casos como no poder memorizar vocabulario, no saber cómo estudiar, entre otros. Parece pues que la categoría es coherente con investigaciones previas, aunque en este caso los ítems sean estrictamente relativos al léxico. En este caso, y en línea también con teorías psicológicas sobre motivación, el hecho de sentir logro (Atkinson y Raynor, 1974) y sentir que son capaces de aprender (Bandura, 1997) redunda en mayores niveles de motivación entre los estudiantes. No obstante, en el caso de no darse esa sensación de éxito y de eficacia personal, como reportan nuestros informantes, la situación deriva en el proceso contrario: la aparición de episodios de desmotivación. 
El segundo factor fue denominado Materiales de clase (Las actividades de vocabulario no me parecen divertidas, Las actividades de vocabulario no me hacen sentir que aprendo, Hago demasiadas tareas de vocabulario que no me parecen útiles). Esta etiqueta ya fue empleada también por Kikuchi (2015) para recoger comentarios como temas no interesantes, textos muy largos, demasiados deberes, entre otros. Nos interesa especialmente el hecho de que dentro de su etiqueta Class materials hay un subapartado (5c) para vocabulario en los materiales. En su caso destaca la desmotivación creada por no saber el vocabulario. Nuestra investigación vendría a complementar los posibles factores desmotivadores relativos al léxico: falta de relevancia personal, falta de sensación de eficacia, falta de interés. Todas estas sensaciones negativas -que de nuevo tienen su correlato en las teorías psicológicas de la motivación- minan poco a poco el compromiso constante que es necesario para aprender.

\section{DISCUSIÓN DE LOS RESULTADOS}

A la luz de los factores atestiguados como desmotivadores en el análisis de los informes y en el análisis factorial, es posible afirmar que el alumnado adulto de ELE experimenta desmotivación específicamente provocada por el aprendizaje de vocabulario. Respondemos así la primera pregunta de investigación.

Además, durante el análisis se categorizaron los siguientes factores: profesorado, exámenes, dificultad del vocabulario, la didáctica del léxico, factores psicológicos y afectivos, experiencias de aprendizaje y materiales de clase. Con este listado respondemos la segunda cuestión de la investigación que era describir en particular los posibles aspectos potencialmente desmotivadores para tener un primer acercamiento, provocar la reflexión tanto investigadora como docente.

Este proceso de reflexión nos lleva a llamar la atención sobre los siguientes aspectos de cara a mejorar la didáctica de ELE que era el objetivo último de este trabajo. En primer lugar, es necesario ser consciente de la trascendencia de los aspectos emocionales, ya que cuando se aprende una lengua extranjera hay demasiada información que retener y la energía empleada puede parecer excesiva en comparación con la percepción que se tiene sobre los resultados alcanzados. En otras palabras, el 
alumnado puede sentir que no está aprendiendo porque no siente el logro o porque se siente desbordado como se puede observar en las siguientes respuestas abiertas.

Pero, a mi parecer, es desfavorable para la comprensión si estudio demasiadas palabras nuevas una vez (Informante 10).

2) Pero tengo muchas palabras que recordar cada día, además de hacer deberes, repasar las lecciones estudiadas, prepararme para las nuevas. Creo que he gastado más tiempo en las palabras, pero no tengo un buen éxito. Esto me desespera. Entonces algunas veces me da angustia aprender el léxico (Informante 14).

3) Cuando estudio las palabras nuevas, me resulta difícil recordar tantos usos diferentes que siempre se los equivoco (Informante 17).

Siempre me vuelvo loca que un texto tenga muchas palabras desconocidas (Informante 46).

Por ello es crucial marcar objetivos claros de lo que se espera que se aprenda de manera que los estudiantes centren sus esfuerzos cognitivos y atención en lo que realmente el docente considera fundamental que interioricen. Siguiendo la teoría de Locke y Latham (1990) este establecimiento de metas ayudará a perseverar en la empresa del aprendizaje. Para ello el docente debe sentar unos objetivos léxicos razonables de manera explícita y dedicar momentos específicos de cada sesión a fomentar la sensación de éxito por haber logrado los objetivos. Con esto, también se cubrirá otra necesidad del ser humano: sentir que obtenemos logros (Atkinson y Raynor, 1974). Por esta razón, sería recomendable, por ejemplo, al comienzo de cada sesión de clase marcar en la pizarra el tema del que se va a tratar. Durante la lección el o la docente dejará claro qué 5 o 6 unidades léxicas son las más importantes; se asegurará de que haya suficiente exposición al input léxico de manera repetida y espaciada a través de diversas modalidades (comprensión auditiva, interacción oral, comprensión lectora, producción escrita...) y al final de la clase junto con la participación de los estudiantes las escribirá en el encerado debajo del tema que era objetivo de la clase (¿Qué palabras hemos dicho boy que eran importantes? Debe darse un refuerzo positivo verbal cuando se acuerden de ellas: ;Genial, bravo! ¡Nos acordamos! ;Cuánto hemos aprendido hoy!). Esta técnica también redundará en la sensación de eficacia percibida y en la autoestima como aprendientes que es fundamental como se ha visto para garantizar una actitud positiva a lo largo del tiempo.

Por otra parte, es importante fomentar un aprendizaje activo donde el estudiantado decida también qué léxico es relevante para su aprendizaje -además del 
seleccionado por el docente-, dejándoles por ejemplo la opción de añadir otro léxico que les gustaría aprender en cuanto a ese tema, otras palabras que creen que van a necesitar..., ya que esto ayuda a parte del estudiantado a mantener el interés.

Me gustan las actividades sobre el léxico que puedo hablar con el profesor. No me gustan demasiado ejercicio del léxico. Aprendo mucho léxico si el léxico tiene gran vinculación con nosotros (Informante 39).

Fomentar este aprendizaje más activo ayuda a que este sea más placentero (Zull, 2002) y, por ende, que se persevere en él. De este modo, se pueden intercalar preguntas del estilo "¿Qué otras palabras creéis que podemos necesitar en esta situación?, ¿Qué otras palabras queréis aprender sobre este tema?”. Al final de la sesión se escribirán las más aclamadas junto con las 5 o 6 seleccionadas por el docente y haciendo hincapié en que el aprendizaje del léxico es muy personal y que cada uno puede gestionar su propio contenido una vez logrados los mínimos establecidos por el nivel objeto de estudio.

Esta estrategia sirve para atajar también la sensación de que aprender léxico es difícil porque se olvida, dado que, al establecerlo claramente, ayudar a la sensación de logro, repetir el input un número mínimo de veces y recuperarlo, dar la libertad de elección de aquel que perciben como más útil para su vida o contexto y recuperarlo en el futuro en otras lecciones se logra evitar testimonios como los siguientes

Pero me resulta molesta que después de un cierto tiempo vaya a olvidar el léxico. Supongo que es que no lo repaso a tiempo y regularmente (Informante 2)

Sin embargo, mucho vocabulario nuevo, especialmente aquello no solemos utilizar en la vida cotidiana y en el trabajo, se utiliza pocas veces y se me olvida rápidamente (Informante 59).

Esto es, la planificación de la enseñanza del vocabulario tiene que tener en cuenta tanto los factores cognitivos, como los afectivos, motivacionales, sociales y culturales para obtener un resultado lo más ajustado posible al aquí y al ahora de cada estudiante.

Por supuesto, para lograr este nivel de individualización es fundamental también hacer explícito el trabajo sobre las estrategias de aprendizaje en el aula que, además, repercuten en la sensación de eficacia personal (Bandura, 1997). Para ello es necesario proporcionar ejemplos de estrategias cognitivas para la comprensión, la clasificación y la memorización durante las clases, así como metacognitivas para ayudarles a planificar su aprendizaje de diferentes formas. 
Las cosas me resultan más desmotivadoras de aprender el léxico es lo que necesito aprender unas palabras de memoria sin la ayuda de una canción, una película, un juego, etc. (Informante 28).

Por supuesto, estas estrategias deben ir acompañadas de las mayores oportunidades sociales posibles de comunicarse y exponerse al input incluso en contextos de no inmersión.

Las tareas del vocabulario no me parecen muy útiles porque no tengo mucha oportunidad de ponerlo en práctica (Informante 9)

Por último, las estrategias emocionales también deben hacerse patentes y comentarse en el aula con el fin de que cada cual encuentre su mejor forma de aprender o, al menos, la más placentera.

(10) Aprendo mucho léxico si el texto es muy interesante, porque si las cosas que estoy estudiando son aburridas, no tengo ganas de seguirlo (Informante 26).

Claro está, estas estrategias y técnicas didácticas que hemos ido desgranando y que son recomendables para evitar la desmotivación de los estudiantes durante el aprendizaje de léxico, deben combinarse y adaptarse a cada contexto. Para ello cada docente debe desarrollar formas de recoger retroalimentación sobre el proceso para ver qué se puede mejorar.

\section{CONCLUSIONES}

La desmotivación es un fenómeno ampliamente documentado en la investigación sobre el aprendizaje de lenguas extranjeras en múltiples contextos y lugares del mundo. De hecho, estos episodios de pérdida de interés suelen tener un origen directo en el sistema y en la forma de aprenderlas: la instrucción formal. Por esa razón, y por el gran interés por el léxico sobrevenido en las últimas décadas tanto en la investigación en lingüística aplicada como en la didáctica de LE, hemos considerado fundamental reflexionar sobre qué aspectos relacionados con la enseñanza del vocabulario pueden provocar la desmotivación con el fin de desarrollar recomendaciones y estrategias didácticas que la minimicen o eliminen.

Por ello nuestras preguntas de investigación eran si, en primer lugar, existe desmotivación provocada por el aprendizaje de léxico y, en segundo lugar, qué factores concretos la desencadenaban. Después del análisis en clústeres temáticos de las 
preguntas abiertas y del análisis factorial de los 15 ítems de Likert se determinaron los siguientes factores desmotivadores: profesorado, exámenes, dificultad del vocabulario, la didáctica del léxico, factores psicológicos y afectivos, experiencias de aprendizaje y materiales de clase.

El objetivo último de ese trabajo era provocar una reflexión crítica de los docentes sobre la enseñanza y con los resultados logrados ofrecer algunas recomendaciones de buenas prácticas didácticas acordes con los resultados documentados.

En suma, en este trabajo se ha ofrecido un estudio exploratorio de la desmotivación que se experimenta cuando los aprendices de ELE se enfrentan al aprendizaje de léxico. Se ha constatado la importancia de los factores afectivos y motivacionales como especialmente sobresalientes, pero sin olvidar que la buena planificación didáctica con resultados exitosos incluye también una reflexión certera sobre aspectos cognitivos, sociales y culturales. Todo ello en conjunto creará un ambiente de aprendizaje más eficaz, seguro y positivo en lo general y también en lo concerniente al aprendizaje de léxico.

\section{BIBLIOGRAFÍA}

Afrough, T., Rahimi, A. y Zarafshan, M. (2014). Foreign Language Learning Demotivation: A Construct Validation Study. Procedia - Social and Behavioral Sciences, 136, pp. 49-53. https://doi.org/10.1016/j.sbspro.2014.05.286

Arai, K. (2004). What 'demotivates' language learners? Qualitative study on demotivational factors and learners' reactions. Toyo Gakuen Daigaku Kiyo, 12, pp. 39-47.

Arefinezhad, H. y Golaghaei, N. (2014). Investigating EFL Learners' attitudes towards demotivating factors in vocabulary learning: a mixed method study. International Journal of Language Learning and Applied Linguistics World, 7 (1), pp. 179-197. https://bit.ly/2zQll7K

Atkinson, J. y Raynor, J. (1974). Motivation and achievement. Washington: Winston \& Sons.

Baba Khouya, Y. (2018). Students Demotivating Factors in the EFL classroom: The Case of Morocco. Advances in Language and Literary Studies, 9(2), pp. 150-159. https://doi.org/10.7575/aiac.alls.v.9n.2p.150

Baldauf, R.; Kaplan, R.B.; Kamwangamalu, N. y Bryant, P. (2011). Success or failure of primary/second foreign language programmes in Asia: what do the data tell us? Current issues in Language Planning, 12(2), pp. 309-323. https://doi.org/10.1080/14664208.2011.609715

Bandura, A. (1997). Self-efficacy: The exercise of control. Nueva York: Freeman.

Barcroft, J. (2015). El método IBI en la enseñanza del léxico: teoría, investigación y nuevas perspectivas. Journal of Spanish Language Teaching, 2, $\quad$ pp. 112-125. https://doi.org/10.1080/23247797.2015.1105512 
Boonchuayrod, P. y Getkham, K. (2018). Why Were English Major Students Demotivated in English Language Learning? International Journal of Management and Applied Science, 4(3), pp. 51-55. https://bit.ly/2ElEOkx

Brown, J. D. (2001). Using surveys in language programs. Cambridge: Cambridge University Press.

Bunyamethi, J. (2018). Los factores desmotivadores en los alumnos tailandeses para el aprendizaje de español como lengua extranjera. Monográfico de SinoELE, 17, pp. 188-202.

Chambers, G. (1993). Taking the 'de' out of demotivation. The Language Learning Journal, 7(1), pp. 13-16. https://doi.org/10.1080/09571739385200051

Comisión Europea (2011). European Strategic Framework for Education and Training (ET 2020) Language Learning at Pre-primary School Level: making it efficient and sustainable a policy bandbook. https://ec.europa.eu/education/sites/education/files/document-library-docs/early-languagelearning-handbook_en.pdf

Consejo de la Unión Europea (2008). Resolución del Consejo de 21 de noviembre de 2008 relativa a una estrategia europea en favor del multilingüismo. https:// bit.ly/2QIbgn9

Department of Education of the Republic of Philippines (2012). Special Program in Foreign Language (SPFL). https://bit.ly/2GfKMWe

Dörnyei, Z. (2001). Teacbing and Researcbing Motivation. Nueva York: Pearson Education.

Dörnyei, Z.; Ryan, S. (2015). The Psychology of the Language Learner Revisited. Nueva York: Routledge. https://doi.org/10.4324/9781315779553

Falout, J. y Maruyama, M. (2004). A comparative study of proficiency and learner demotivation. The language teacher, 28 (8), 3-9.

Falout, J. y Falout, M. (2005). The other side of motivation: Learner demotivation. En K. BradfordWatts, C. Ikeguchi y M. Swanson (Eds.), JALT2004. Conference proceedings. Tokio: JALT.

Falout, J. (2012). Coping with demotivation: EFL learners' remotivation processes. The Electronic Journal for Teaching English as a Foreign Language, 13(3), pp. 1-29.

Field, A. (2005). Discovering statistics using SPSS (2nd ed.). London: Sage.

Gottlieb, N. (2011). Japan: Language Policy and Planning in Transition. Current Issues in Language Planning, 9(1), pp. 1-68. https://doi.org/10.2167/cilp116.0

Hair, J.F., Anderson, R.E., Tatham, R.L. y Black, W.C. (1999). Análisis Multivariante. Madrid: Prentice Hall.

Hamada, Y. y Grafström, B. (2014). Demotivating factors in learning Japanese as a foreign language. 秋 田大学教育基礎教育研究年報, pp. 9-14. https://bit.ly/2Utm8Ez

Hasegawa, A. (2004). Student demotivation in the Foreign Language Classroom. Takushoku Language Studies, 107, pp. 119-136.

Hu, R. J. S. (2011). The relationship between demotivation and EFL Learners' English Language Proficiency. English Language Teaching, 4(4), pp. 88-96. https://doi.org/10.5539/elt.v4n4p88

Hu, Y. (2007). China's foreign language policy on primary English education: What's behind it? Language Policy, 6(3-4), pp. 359-376. https://doi.org/10.1007/s10993-007-9052-9 
Kaiser, H.F. (1958). The Varimax Criterion for Analytic Rotation in Factor Analysis. Psychometrika 23(3), 187-200. https://doi.org/10.1007/BF02289233

Kaivanpanah, S. y Ghasemi, Z. (2011). An investigation into Sources of demotivation in second language learning. Iranian Journal of Applied Linguistics, 14(2), pp. 89-110.

Kaplan, R. B.; Baldauf Jr.; Richard B. y Kamwangamalu, N. (2011). Why educational language plans sometimes fail. Current issues in Language planning, 12(2), pp. 105-124. https://doi.org/10.1080/14664208.2011.591716

Keblawi, F. (2005). Demotivation among Arab learners of English as a foreign language. En M. Singhal y J. Liontas (Eds.). Proceedings of the second international online conference on second and foreign language teaching and research. The reading matrix. Estados Unidos. https://bit.ly/2SyYG6Y

Kikuchi, K. y Sakai, H. (2009). Japanese Learner's demotivation to Study English: a Survey study. JALT Journal, 31(2), pp. 183-203. https://bit.ly/2G5oeHt

Kikuchi, K. (2015). Demotivation in Second Language Acquisition. Insights from Japan. Bristol: Multilingual Matters. https://doi.org/10.21832/9781783093953

Kim, T. Y. y Kim, Y. K. (2015). Elderly Korean Learners' participation in English Learning through lifelong education: Focusing on motivation and demotivation. Educational Gerontology, 41, pp. 120-135. https://doi.org/10.1080/03601277.2014.929345

Lewis, M. (1993). The lexical Approach. The State of ELT and a Way Forward. Boston: LTP.

Laufer, B. (2005). Focus on Form in Second Language Vocabulary Learning. EUROSLA Yearbook • January 2005, pp. 223-250. https://doi.org/10.1075/eurosla.5.11lau

Li, L. y Zhou, C. (2013). Different faces of Demotivation: A comparative study on Chinese and Korean college EFL Learners' demotivators. Journal of Applied Sciences, 13, pp. 800-809. https://doi.org/10.3923/jas.2013.800.809

Locke, E.A. y G.P. Latham. 1990. A Theory of goal setting and task performance. Englewood: Prentice Hall.

Méndez Santos, M. C. (2019). Estudios sobre la desmotivación del alumnado en el aprendizaje formal de lenguas extranjeras: estado de la cuestión. Études romanes de Brno, 40(1), pp. 99-122. https://doi.org/10.5817/ERB2019-1-7

Méndez Santos, M. C. (2020). La desmotivación de los estudiantes adultos de ELE: un acercamiento desde los sistemas dinámicos complejos. En A. León-Manzanero e I. Mañas Navarrete (Eds.). Enseñar y aprender español para la comunicación intercultural. Culture Crossroads, 15 (número monográfico).

Muhonen, J. (2004). Second language demotivation: Factors that discourage pupils from learning the English language. Trabajo de fin de grado, University of Jyvaskyla, Finlandia.

Muthen, B. y Kaplan, D. (1992). A comparison of some methodologies for the factor analysis of nonnormal Likert variables: A note on the size of the model. British Journal of Mathematical and Statistical Psychology, 45(1), pp. 19-30. https://doi.org/10.1111/j.2044-8317.1992.tb00975.x

Nation, P. (2001). Learning Vocabulary in another language. Cambridge: CUP. https://doi.org/10.1017/CBO9781139524759 
Nguyen, H. B. y Thi Pham, V. T. (2017). English-major students' perceptions of demotivating factors in learning reading. International Journal of advanced research, 5(8), pp. 200-207. https://doi.org/10.21474/IJAR01/5051

Olsson, U. H., Foss, T., Troye, S. V. y Howell, R. D. (2000). The performance of ML, GLS, and WLS estimation in structural equation modeling under conditions of misspecification and nonnormality. Structural equation modeling, 7(4), pp. 557-595. https://doi.org/10.1207/S15328007SEM0704 3

Perry, F. (2017). Research in Applied Linguistics. Nueva York: Routledge. https://doi.org/10.4324/9781315394664

Rasinger, S. (2019. La investigación cuantitativa en lingüistica. Una introducción. Madrid: Akal.

Richards, J. y Rodgers, T. (2003). Enfoques y métodos en la enseñanza de idiomas. Madrid: Cambridge University Press.

Shu-Chen, H. (2012). Pushing learners to work through tests and marks: Motivating or demotivating? A case in a Taiwanese University. Language Assessment Quarterly, 9(1), pp. 60-77. https://doi.org/10.1080/15434303.2010.510898

Tabachnick, B. G. y Fidell, L. S. (2007). Using multivariate statistics (5th ed.). Boston: Pearson Education.

Thornbury, S. (2002). How to teach vocabulary. Essex: Pearson Education.

Xuezheng, J. (2014). Investigation of Learner Demotivation in English Learning in Chinese College. International Journal on Studies in English Language and Literature, 2(12), pp. 39-45.

Zull, J. (2002). The art of Changing the Brain, Enriching the Practice of Teaching by Exploring the Biology of Learning. Virginia: Stylus. 